SAND77.8755

Unlimited Release
Publications and Public

Information Division, 8265

for TIC

(2)

\title{
Monte Carlo Studies of Sputtering
}

(Presented at the Third International Conference on Plasma Surface Interactions in Controlled Fusion Devices, Culham Laboratory, UK, April 3-7, 1978 and to be published in the Journal of Nuclear Materia/s)

L. G. Haggmark, W. D. Wilson 


\section{MONIE CARLO STUDIES OF SPUTTERTING * \\ L. G. Haggmark and W. D. Wilson \\ Sandia Laboratories \\ Livermore, California}

\section{ABSTRACT}

A conputer program has been developed to simulate the sputtering process using the Monte Carlo method and the binary collision approximation. This program is a result of the generalization of the TRTM computer program such that the target atam trajectories are followed in addition to those of the incident particles. This program, which includes elatronic energy loss, uses an analytic formula which is based on realistic interatomic potentials for detemining particle scattering angles and the energy transfer to target atams. A model of the sputtering process has been developed for physically defining the surface and bulk binding energies necessary for calculations. A number of sputtering yield calculations have been performed for $\mathrm{H}, \mathrm{D}, \mathrm{T}$, and ${ }^{4} \mathrm{He}$ ions incident on $\mathrm{C}, \mathrm{Ni}, \mathrm{MO}$, and $\mathrm{Au}$ targets for energies less than $10 \mathrm{keV}$. The valiuity of the Monte Carlo model is demonstrated by the good agreement between the calculated results and the most recent experiments.

*Work supported by the Division of Magnetic Fusion Energy of the Department of Energy under Contract Number AT (29-1)-789. 


\section{Introduction}

Plasma contamination by sputtered wall material is one of the crucial problens for present plasma experiments and future fusion reactors [1]. The sputtering is caused primarily by low energy, light ions and neutrals from the plasma. Measurements of the small sputtering yields for light particles are inherently difficult, and sputtering theories $[2,3]$ are still not accurate enough to predict absolute values at low energies [4]. To provide some futher insight into the sputtering mechanisms, a Monte Carlo method using the binary collision approximation has been applied to simulate the sputtering process.

Similar methods have been applied previously [5-8] tr simulate sputtering, but the majority of the reported results [5-7] have been for heavier incident particles. Only the recently presented results of Maderlechner et al [8], using the MARLOWE [9] computer program, have dealt with light particle sputtering. Here we have used a generalization of the TRMM [10] computer program which treats particle transport in amorphous solids. This program, which includes electronic energy loss, uses an analytic formula $[10,11]$ which is based on realistic interatanic potentials for detemining particle scattering angles and the concamitant energy transfer to target atoms. Thus, it is both fast, in terms of computer usage, and accurate within the constraints of the binary collision approximation.

A simplified model of the sputtering processes has been developed which is applicable to the Monte Carlo method. Parameters of this model are physically meaningful and are related to "surface" and "bulk" binding energies of the target atams. A muber of calculations have been performed for $\mathrm{H}, \mathrm{D}, \mathrm{T}$, and ${ }^{4} \mathrm{He}$ ions incident on $\mathrm{C}, \mathrm{Ni}, \mathrm{MO}$, and Au targets for energies less than $10 \mathrm{keV}$. Comparisons are made between our computed results and those of the most recent experiments. 


\section{Monte Carlo Procedure and Sputtering Mbdel}

To simulate the sputtering process, the TRIM Mante Carlo computer program has been generalized to consider the trajectories of the target atams as well. as those of the incident ion. The full details of the TRTM computer program have been described elsewhere [10]. In brief, each particle starts with a given energy, position, and direction, and its trajectory is followed in a target, collision by collision, until its energy falls below a prespecified value or until it leaves the target surface. The particle changes direction as a result of binary muclear (elastic) collisions and moves in a straight path between collisions. The particle's energy is reduced by nuclear and electronic (inelastic) energy losses, and these energy loss mechanisms are assumed to be independent. The target is assumed to be amorphous (atoms at random locations) so that the directional properties of a crystal lattice are ignored. The low energy, electronic energy loss $\Delta \mathrm{E}_{e}$ is based on the velocity dependent treatment of Lindhard and Scharff [12]. Thus, $\Delta \mathrm{E}_{\mathrm{e}}=\mathrm{LNK} \sqrt{\mathrm{E}}$, where $\mathrm{L}$ is the pathlength between collisions, $\mathrm{N}$ is the target atanic density, $\mathrm{k}$ is a proportionality constant, and $\mathrm{E}$ is the particle's energy. For the results presented here, we have used the Iindhand and Scharff [12] expression for $k$ and with $L=N^{-1 / 3}$. Although it is reoognized that deviations from this electronic energy loss treatment are known to exist [10], the sensitivity of the sputtering yields to variations from this treatment has not been investigated in these studies. Such variations would effect primarily the transport of the incident light particles, since the energy loss of the low energy target atams is dominated by the nuclear energy loss mechanism.

For the nuclear energy loss $\mathrm{T}$ and the associated scattering angle, use is made of a parameterized analytic fonmla derived by Biersack et al. [10, 11]. This formula very accurately describes classical scattering for any 
repulsive interatomic potential given the particle's energy, impact parameter, and distance of closest approach. The values for the parameters in the scattering formula were determined by a least-squares fitting procedure using precomputed, mumerical evaluations of the classical scattering integral [13] based on the Moliere [14] potential. This potential with the Firsov [15] screening length is used for most routine calculations with the TRIM program.

one of the more important attributes of this Monte Carlo approach is that the most realistic interatomic potentials can be readily used. Here, we have calculated the various atcm-atom potentials that were necessary using the freeelectron approximation as described by Wilson and Bisson [16]. To facilitate the use of these potentials, we adjusted the screening length, in most cases, in the Moliere screening function to give the best fit to the free-electron potentials. For the $\mathrm{MO}^{+}-\mathrm{MO}^{+}, \mathrm{Au}^{+}-\mathrm{Au}^{+}$, and $\mathrm{H}-\mathrm{C}$ potentials a better fit was acrieved by adjusting the screening length $\alpha$ in the following screening function: $\Phi(r / \alpha)=0.100 \mathrm{e}^{-0.22 \mathrm{r} / \alpha}+0.472 \mathrm{e}^{-0.50 \mathrm{r} / \alpha}+0.428 \mathrm{e}^{-1.51 \mathrm{r} / \alpha}$, where $r$ is the interatomic separation. This screening function was presented by wilson et al. [17] in reporting their results for $\mathrm{NO}^{\circ}-\mathrm{N} \mathrm{b}^{\circ}$ potential using the free-electron method. The screening lengths used to fit the screening functions to the potentials are given in Table I together with those of Firsov. As mentioned above, the impact parameter and the distance of closest approach, in addition to the energy, are needed to use the nuclear scattering formalism. With the pathlenyth between collisions being $\mathrm{N}^{-1 / 3}$ the impact parameter $P$ is randamly selected using the following formula:

$$
P=\left[R_{n} / \pi N^{2 / 3}\right]^{] / 2}
$$


where $R_{n}$ is a randam number uniformly distributed between 0 and 1 . This assumes there is one atom in the volume element of length $N^{-1 / 3}$ and base area $\mathrm{N}^{-2 / 3}$. This procedure maintains the atomic density in the target but ignores any correlation between the positions of successive atams. The tistance of closest approach is then obtained, in the usual manner, from the pole of the integrand in the classical scattering integral.

In generalizing the TRM formalism to follow the target atom trajectories and their subsequent possibility of becaning sputtered atams, we have set certain criteria for considering the motion and escape of these atams. This part of the procedure fullows closely that defined for the general displacement model in the MARLOWE [9] program. A particle with original kinetic energy E emerges fram a nuclear collision with kinetic energy $E_{1}$, after transferring energy $T$ to a target atam, i.e., $E_{1}=E-T$. The target atam's motion is considered if the energy $\mathrm{T}$ is greater than a threshold energy $\mathrm{E}_{\mathrm{d}^{\prime}}$ and if $T>E_{\mathrm{d}^{\prime}}$ it then loses some binding energy $\mathrm{F}_{\mathrm{b}} \leq \mathrm{E}_{\mathrm{d}}$. Thus, the final kinetic energy $\mathrm{E}_{2}$ of a target atcm is glven by $\mathrm{E}_{2}=\mathrm{T}-\mathrm{E}_{\mathrm{b}}$. In the calculations presented here we have set $E_{\mathrm{d}}=\mathrm{E}_{\mathrm{b}}$, and the value of $\mathrm{E}_{\mathrm{b}}$ depends on whether the collision is with a surface or a bulk atan. In what follows, a model for physically defining the $\mathrm{F}_{\mathrm{b}}$ values is presented.

An ion incident upon a metal surface transfers energy to both the surface and bulk atams of the metal in its slowing down process. This transferred energy may result in the production of surface and bulk vacancies and vacancy clusters as well as the associated interstitial loops. In order to bring a measure of physical understanding to the Nonte Carlo calculation presented here and also to provide a predictive capability, a simplified model of these camplex radiation damage processes has been developed. 
In this model, the incident ions may transfer energy to the "surface" and "bulk" atams, these two types being the cnly ones distinguished between. Atams lying between vacum and $1 / 4$ a (a - lattice parameter) of the surface are considered "surface" atoms and all others are taken to be "bulk." Very real surface complications such as angular orientation, steps, kinks, jogs and effects due to impurities are neglected. Similarly, atoms quite near the surface, i.e., within a few monolayers, are approximated as bulk atans.

The shall consider the bulk atoms first. The energy required to remove a bulk atan to infinity is denoted $\mathrm{E}_{\mathrm{V}}^{\infty}$ and is given by:

$$
E_{V}^{\infty}=E_{l V}^{F}+E_{\text {HS }}
$$

where $E_{1 V}^{F}$ is the vacancy formation energy and $E_{\text {HS }}$ the heat of sublimation of the solid; $\overrightarrow{F V}$ is the energy required to place a lattice atom on the surface (i.e., to bring it first to infirity and then back to the surface); $\Sigma_{\mathrm{HS}}$ is the energy per aton to remove an entire layer of the surface to infinity and there dissociate all the atams of the layer. In forning a bulk vacancy, a lattice atom is brought to infinity and then placed back into an interstitial site, forming a Frenkel pair. The "binding" energy of a lattice atan, $\mathrm{E}_{\mathrm{b}}$ ' is therefore given by:

$$
E_{b}=E_{V}^{\infty}+E_{I}^{F}
$$

where $E_{I}^{F}$ is the self-interstitial fonmation energy.

It is reoognized that in using Eq. (4) for the binding energy, one is neglecting the dynamics of the process going on. That is, the ejected lattice atom may not actually be in an interstitial position but may create further 
knock-ons, and may find itself finally in a substitutional site. Even if it is in an interstitial configuration, that configuration may not be the lowest energy one because the lattice relaxations may not yet have equilibrated. We therefore propose using the equation:

$$
E_{b}=E_{V}^{\infty}+E_{I}^{\prime}
$$

defining $E_{I}^{*}$ as an effective interstitial fomation energy. Energy $E_{b}$ is subtracted from the transferred energy $T\left(=\gamma E \sin ^{2} \theta / 2\right)$ to give the kinetic energy of the ejected particle.

Before turning to the surface aum binding, let us be more specific about the volume dependence of the terms already defined. The vacancy formation energy can be written:

$$
E_{1 v}^{F}=-\sum_{j} v\left(r_{v j}^{b}\right)+\sum_{j} v\left(r_{v j}^{s}\right)+E_{v o l}
$$

where $\mathrm{V}\left(\mathrm{r}_{\mathrm{vj}}^{\mathrm{x}}\right)$ is the interatanic potential between a lattice atam at the vacant site in the buik $(x=b)$ or on the surface $(x=s)$. (For a first-neighbor two-body potential having magnitude $\phi$ at the first-neighbor separation, $\sum_{j} v\left(r_{i j}^{b}\right)$ $=12 \phi$ and $\left.\sum_{j} V\left(r_{v j}^{S}\right)=6 \phi.\right)$ Similarly, the heat of sublimation can be written:

$$
E_{H S}=-\sum_{j} v\left(r_{v j}^{s}\right)-E_{v o l}
$$

In Egs. 6 and 7, $E_{\mathrm{vol}}$ is the volume dependent part of the energy, that is, that part of the energy of the lattice which cannot be represented by twobody forces [18]. From Egs. 6 and 7 , 


$$
E_{\text {vol }}=\frac{1}{2}\left(E_{1 V}^{F}-E_{\text {HS }}\right)
$$

We now define $\mathrm{E}_{\mathrm{s}}^{+}$to be the energy required to remove a surface atom to infinity and $\mathrm{E}_{\mathrm{s}}^{-}$the energy required to remove a suriace atom and place it in the bulk. Clearly,

$$
E_{S}^{-}=E_{S}^{+}+E_{I^{-}}^{-}
$$

we write

$$
E_{S}^{+}=\mathrm{fE}_{\mathrm{V}}^{\infty}-E_{\mathrm{Vol}},
$$

whene $f$ is fraction of the (volume independent) energy required to bring an atom from the bulk to infinity which must be expended to bring an atam fram the surface to infinity. Using Egs. 3 and 8 in Eg. 10, it is easy to show that

$$
E_{S}^{+}=E_{H S}+\left(f-\frac{1}{2}\right)\left(E_{1 V}^{F}+E_{H S}\right)
$$

Simple estimates of $f$ can be made by comparing the number of nearest neighbor bonds on a surface $\left(\mathrm{N}_{\mathrm{s}}\right)$ to the number in the bulk $\left(\mathrm{N}_{b}\right)$. For a foc material, the $(100)$ surface gives $\left(\mathrm{N}_{\mathrm{s}} / \mathrm{N}_{\mathrm{b}}\right)=f=2 / 3$, while for a (111) surface $f=3 / 4$. The important point to be made is that for any value of $f>1 / 2$, it takes more energy $\left(\mathrm{E}_{\mathrm{S}}^{+}\right.$) than the heat of sublimation $\left(\mathrm{E}_{\mathrm{HS}}\right)$ to rercive a surface atom to infinity. These arguments are consistent with the experimental results of Bay et al. [19] who find that the threshold anergies for sputtering appear to be higher $t:$ an that dictated by the heat of sublimation.

To summarize, if a collision occurs on the surface (depth $\leq 1 / 4$ a) then $E_{b}=E_{s}^{+}$for a target atom which will leave the surface on its initial free 
flight path $\mathrm{L}$ and $\mathrm{E}_{\mathrm{b}}=\mathrm{E}_{\mathrm{S}}^{-}$for an aton that will have a collision on its iritial path. At depths greater than $1 / 4 a$,

$$
E_{b}=E_{I V}^{F}+E_{H S}+E_{I}^{\prime}
$$

The values of $\mathrm{E}_{\mathrm{HS}}$ and $\mathrm{E}_{\mathrm{IV}}^{F}$ used in the present calculations are given in Table II. Using thuse values, results for ${ }^{4} \mathrm{He}$ nomally incident on Ni are illustrated in Fig. 1 for $E_{I}^{*}=0,1$, and $3 \mathrm{eV}$, and for $f=2 / 3$ and 3/4. Note in this figure that the results are not sensitive '.. the choice of $f$ except at very low energies. A value of $f=3 / 4$ was chosen throughout the remainder of this work.

It should be pointed out that a value of $3 \mathrm{eV}$ for $E_{I}^{\prime}$ is not unreasonable. Johnson and wilson [23] found $E_{I}^{F}$ to be $~ 3.3$ ev using a pair potential and noncentral force for $\mathrm{Ni}$. Absolute values of fomation energies are very difficult to calculate, however, and other pair-potentials may well give widely different resilts. In bcc materials, interstitial fomation energies are usually found to be higher.

\section{Results and Discussion}

For the Monte carlo rapll. Tresented ners, a suificient number of incident particle histori... processed at each energy to yield at least 100 sputtered atons, and in the majority of the cases this number exceeded 200. Comparisons of repeated calculations with different sequences of randam numbers indicated that the statistical errors are approximately 10 to 20 percent. We performed the calculations at energies which correspond closely with those used in the experiments, i.e., $0.05,0.08,0.1,0.15,0.24,0.5$, 1, 2, 4, and $8 \mathrm{kev}$. Most of our comparisons with experimental results are 
with the recent data generated by the Garching group [19, 24, 25]. The reader is referred to their original papers for further cumparisons with previous results.

The calculated sputtering yields (atams/ion) already presented in Fig. 1 indicate that $E_{I}^{\prime}=3 \mathrm{eV}$ is a suitable value fo. $\mathrm{Ni}$ and this value has been used for the other Ni calculations. Fig, 2 wwows the calculated results for $\mathrm{H}, \mathrm{D}, \mathrm{T}$, and ${ }^{4} \mathrm{He}$ nomally incident on $\mathrm{Ni}$ togetiner with the experimental measurements of Bohdansisy et al. [24] for $H, D$, and ${ }^{4} \mathrm{He}$. Our results show the same energy dependent trends as those of the experiments with the maximu yield in the 1 to $3 \mathrm{keV}$ range. Also, near the maximum yield: the ratios of the ${ }^{4} \mathrm{He}$ and $\mathrm{D}$ sputtering yields to that for $\mathrm{H}$ is about the same as determined experimentally. The calculated results at energies above $1 k-v$ are somewhat lower than experiment for ${ }^{4} \mathrm{He}$ and higher for $\mathrm{H}$ and $\mathrm{D}$. Nevertheless, the overall comparison between the calculations and experiments seems encouraging considering our use of the binary collision approximation and the inherent difficulties in measuring small sputtering yields.

Figs. 3 and 4 show our results for D, T, and ${ }^{4}$ He nonmally incident on Au and $\mathrm{M}$, respectively. In these figures, we also show the results of Bay et al. [19] for $D$ and ${ }^{4} \mathrm{He}$. We again used $E_{I}^{-}=3$ eV for $\mathrm{Au}$, but as discussed earlier, this value is expected to be too low for bac wo. We therefore show in Fig. 4, the ${ }^{4} \mathrm{Fe}-\mathrm{No}$ results using $\mathrm{E}_{\mathrm{I}}^{\prime}=3,10$, and $20 \mathrm{eV}$ and show only the $E_{I}^{\prime}=20 \mathrm{eV}$ results for $T$ and $D$. It is felt that $E_{I}^{\prime}=20 \mathrm{eV}$ may be too hijh a value to be considered an interstitial formation energy and its necessity may be due to a breakdown of the binary collision approximations. Molecular dynamical calculations are planned to help determine the sorrce of this large $E_{I}^{\prime}$ valive. 
We determine the sputtered atom energy spectra in our calculations, as well as the angular distributions. The angular distributions are all essentially cosine distributions, as is to be expected fram our Monte Carlo model, since our targets are amorphous and we consider no influense of surface atoms on the sputtered atoms once they leave the surface. An example of the sputtered energy spectrum is shown in Fig. 5 for $1 \mathrm{keV}{ }^{4} \mathrm{He}$ normally incident on Au. The lashed lines in this figure indicate the variation of the energy distribution in terms of the energy power function $\mathrm{E}^{-\mathrm{n}}$ with $\mathrm{n}=1.5,1.8$, and 2.0. Our results indicate that the spectrum is somewhat harder than $E^{-2}$ as predicted by Sigmund [2], but this is consistent with the recent measurements of Huaks et al. [26] for light ions an Au $(n=1.8)$.

Finally, the calculated sputtering yields for $\mathrm{H}, \mathrm{D}$, and $\mathrm{T}$ nomally incident on $C$ are shown in Fig. 6. Also, the results from three recent experiments are included in the figure. We realize that there are many types of "carbon" and their sputtering properties [4] can vary markedly. our purpose here is to show the energy dependence of the sputtering yields predicted by the Monte Carlo formalism using nominal values for $E_{H S}$ and $E_{T V}^{F}$ (see Table I) with $E_{I}^{*}=3 \mathrm{eV}$. The calculated results indicate that the maximm yield occurs in the energy range from about 0.3 to $0.5 \mathrm{keV}$. These results disagree with the sputtering yield energy dependence reported by Smith et al. [27] for $H$, but they are consistent with the measurements of Bohdansky et al. [25] for H and Borders et al. [28] for D.

\section{Acknowledgements}

The authors wish to acknowledge the insight and interest that M. I. Baskes and C. F. Melius, of this Laboratory, gave to the binding energy formalism developed for this paper. Also, we wish to express our appreciation to C. L. 
Bisson, of this Laboratory, for his assistance in the calculations of the free-electron potentials and to $\mathrm{W}$. Bauer for his enco:rragement in this work. 
1. R. Behrisch, Muclear Fusion 22 (1972) 695.

2. P. Sigmand, Phys. Rev. 184 (1969) 383.

3. R. Weissmann and P. Sigmund, Rad. Effects 19 (1973) 7.

4. B. M. U. Scherzf $x$, R. Behrisch and J. Roth, Proc. Int. Symp. on PlasmaWall Interaction, Jïlich (Pergamon Press, Frankfurt, 1977) p. 353.

5. P. V. Pavlov, D. I. Telel'baum, F. I. Zorin, and V. I. Alekseev, Fiz. Tver. Tela $\underline{8}$ (1966) 2679 [Sov. Phys. - Solid sate $\underline{8}$ (1967) 2141].

6. G. Betz, R. Dobrozensky and F. P. Viehböck, Int. J. Mass Spectram. Ion Phys. 6 (1971) 451.

7. I. Ishitani and R. Shimizu, Appl. Phys. 6 (1975) 241.

8. G. Maderlechner, R. Behrisch, B. M. U. Scherzer, and M. T. Fobinson, VIIth International Conference on Atamic Collisions in Solids, Moscow, September 19-23, 1977 (Proceedings to be published by Mbscow State University Publishing House).

9. M. T. Rabinson and I. M. Torrens, Phys. Rev. B9 (1974) 5008.

10. 5. P. Biersack and I G. Haggmark, submitted for publication.

11. J. F. Biersack, I. G. Haggmark, and M. I. Baskes, to be published.

12. J. Iincthand and M. Scharff, Phys. Rev. 124 (1961) 128.

13. M. T. Babinson, publication of the Oak Ridge National Laboratory, Oak Ridge Tennessee, Report ORN-4556 (1970) (umpublished).

14. G. Nolière, 2. Naturforschung. A2 (1947) 133.

15. O. B. Firsov, Zh. Eksp. Teor. Fiz. 33 (1957) 696 (Sov. Phys. - JETP $\underline{6}$ (1958) 534].

16. W. D. Wilson and C. L. Bisson, Phys. Rev. B3 (1971) 3984.

17. W. D. Wilson, L. G. Haggmark, and J. P. Biersack, Phys. Rev., B15 (1977) 2458.

18. R. A. Johnson, Phys, Rev. B6 (1972) 2094.

19. H. I. Bay, J. Roth, and J. Bohdansky, J. Appl. Phys. 48 (1977) 4722. 
20. C. J. Snith (ed.), Metal kefererce Book, 5th edition, Butterworths, London (1976), p. 186 .

21. A. Seeger and H. Mehrer, Vacancies and Interstitials in Metals, edited by A. Seeger, D. Schmacher, N. Schilling, and J. Diehl (North Holland Publishing Co., Amsterdam, 1970) p. 1.

22. M. Doyama and J. S. Koehler, Acta metall. 24 (1976) 871.

23. R. A. Johnson and W. D. Wilson, Interatomic Potentials and simulation of Lattice Defects, edited by P. C. Gehlen, J. R. Beeler, and R. I. Jaffee (Plenum Press, New York, 1972) p. 301.

24. J. Bohdansky, H. L. Bay, and J. Roth, Proc. VIIth Int. Vacuum Congress and IIIrd Int. Conf. on Solid Surfaces, edited by R. Dobrohemsky, F. Rïdenaver, F. P. Viehbröck, and A. Breth (Berger and Söhner, Vienna, 1977), p. 1509.

25. J. Bohdansky, J. Foth, and M. K. Sinha, Proc. 9th Symp. on Fusion Technology, Gamisch (1976) p. 541.

26. P. Hucks, G. Stöcklin, E. Vietzke, and K. Vogelbruch, this conference.

27. J. N. Snith, Jr., C. H. Meyer, Jr., and J. K. Layton, J. Nucl. Mater. 67 (1977) 234 .

28. J. A. Borders, R. A. Langley, and K. L. Wilson, this conference. 
Screening Lengths Used in Fitting the Screening Function of Moliere and Eq. (1) to the Free-Electron Potentials.

\begin{tabular}{|c|c|c|}
\hline \multirow[b]{2}{*}{ Fotential } & \multicolumn{2}{|c|}{ Screening Lengths (rm) } \\
\hline & $\begin{array}{ll}\text { Screening Function } \\
\text { Moliere } & \text { Eq. (1) }\end{array}$ & Firsov \\
\hline $\mathrm{H}-\mathrm{Ni}^{+}$ & 0.0118 & 0.0137 \\
\hline $\mathrm{He}-\mathrm{Ni}^{+}$ & 0.0101 & 0.0132 \\
\hline $\mathrm{Ni}^{+}-\mathrm{Ni}^{+}$ & 0.00819 & 0.00972 \\
\hline $\mathrm{H}-\mathrm{MO}^{+}$ & 0.0113 & 0.0122 \\
\hline $\mathrm{He}-\mathbf{M O}^{+}$ & 0.0103 & 0.0118 \\
\hline $\mathbf{M b}^{+}-\mathbf{M b}^{+}$ & 0.00810 & 0.00849 \\
\hline $\mathrm{H}-\mathrm{Au}^{+}$ & 0.0102 & 0.0102 \\
\hline $\mathrm{He}-\mathrm{Aul}^{+}$ & 0.00928 & 0.00989 \\
\hline$A u^{+}-A u^{+}$ & 0.00675 & 0.00699 \\
\hline $\mathrm{H}-\mathrm{C}$ & 0.00160 & 0.0205 \\
\hline$c-c$ & 0.0128 & 0.0162 \\
\hline
\end{tabular}




\section{TABLE II}

$$
\text { Value for } \mathrm{E}_{\mathrm{HS}} \text { and } \mathrm{E}_{\mathrm{IV}}^{\mathrm{F}}
$$

$\begin{array}{lcc}\text { Element } & E_{H S}(\mathrm{eV})^{(a)} & E_{\text {IV }}^{F}(\mathrm{eV}) \\ \mathrm{Ni} & 4.45 & 1.4^{(\mathrm{b})} \\ \mathrm{Hb} & 6.89 & 2.4^{(\mathrm{c})} \\ \text { Au } & 3.93 & .9^{(\mathrm{b})} \\ \mathrm{C} & 7.38 & (2.5)^{(\mathrm{d})}\end{array}$

(a) Metal Reference Book [20]

(b) A. Seeger and H. Mehrer [2I]

(c) M. Doyama and J. S. Koehler [22]

(d) Assumed 
Fig. I - Mnte Carlo calculations of the sputtering yields for ${ }^{4}$ He normally incident on Ni for energies less than $10 \mathrm{keV}$. The parameters $f$ and $\mathrm{E}_{\mathrm{I}}^{\prime}$ are used in the binding energy formalism (see text). The experimental sputtering yield of Bohdansky et al. [24] are presented for comparison purposes.

Fig. 2 - Calculated sputtering yields for H, D, T, and ${ }^{4}$ He nomally inctdent on $\mathrm{Ni}$ for energies less than $10 \mathrm{keV}$. The experimental sputtering yields of Bohdansky et al. [24] for H, D, and 4 He are presented for comparison purposes.

Fig. 3 - Calculated sputtering yields for $D, T$, and ${ }^{4}$ He nomally incident on Au for energies less than $10 \mathrm{keV}$. The experimental sputtering yields of Bay et al. [19] for $D$ and 4 Be are presented for comparison purposes. Fig. 4 - Calculated sputtering yield for $D, T$, and ${ }^{4}$ He normally incident on Mo for energies less than $10 \mathrm{keV}$. The experimental sputtering yields of Bay et al. [19] for D and ${ }^{4} \mathrm{He}$ are presented for comparison purposes.

Fig. 5 - Energy distribution of the sputtered atoms for $1 \mathrm{keV}{ }^{4} \mathrm{He}$ normally incident on flu. The histogram is the calculated results and the dashed lines indicate the energy power function $\mathrm{E}^{-\mathrm{n}}$ for $\mathrm{n}=1.5,1.8$ and 2.0.

Fig. 6 - Calculated sputtering yields for H, T, and D nomally incident on C for energies less than $10 \mathrm{kev}$. The experimental sputtering yields of Bonders et al. [28], Smith et al. [27], and Bohdansky et al. [25] are presented for comparison purposes. The dashed lines through the experimental $\mathrm{H}$ yields are intended only as a visual guides. 


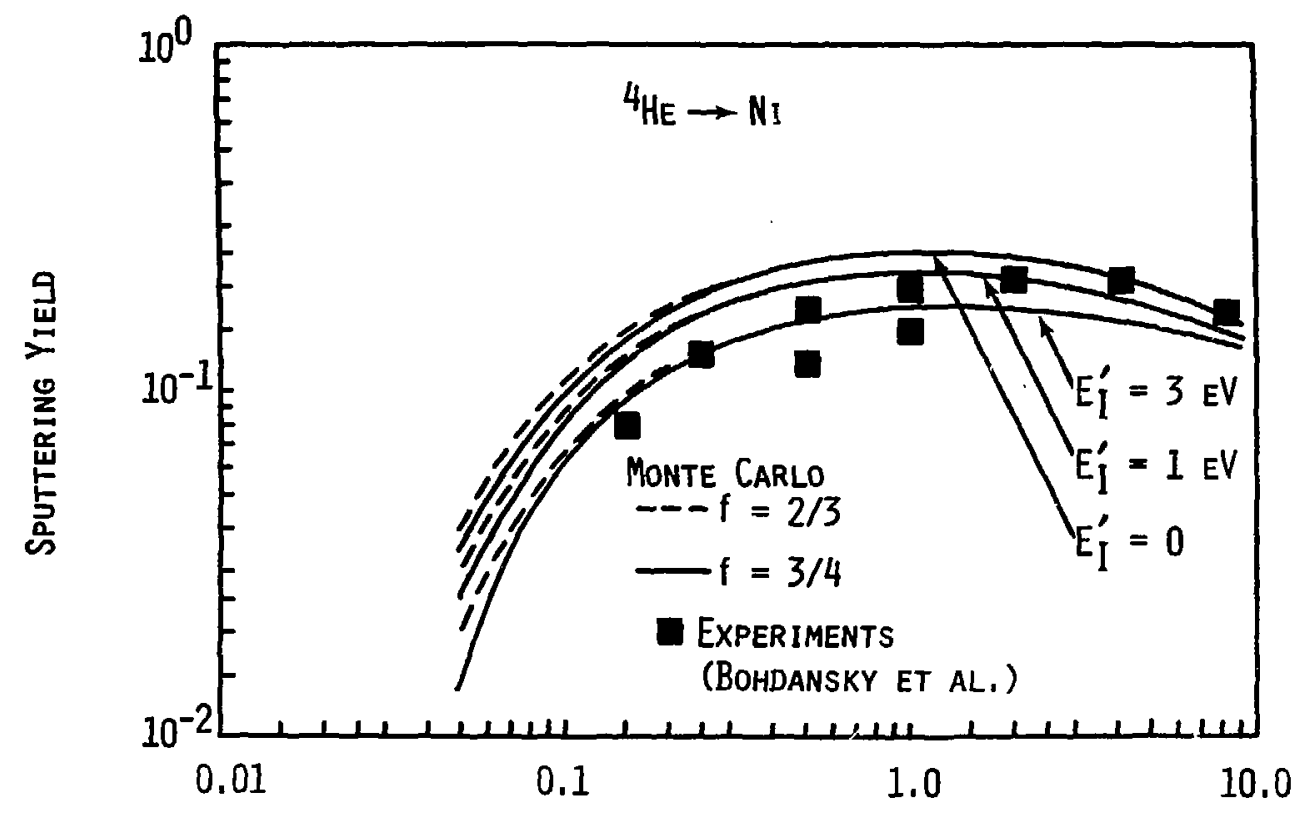

ENERGY (KEV)

Figure 1 


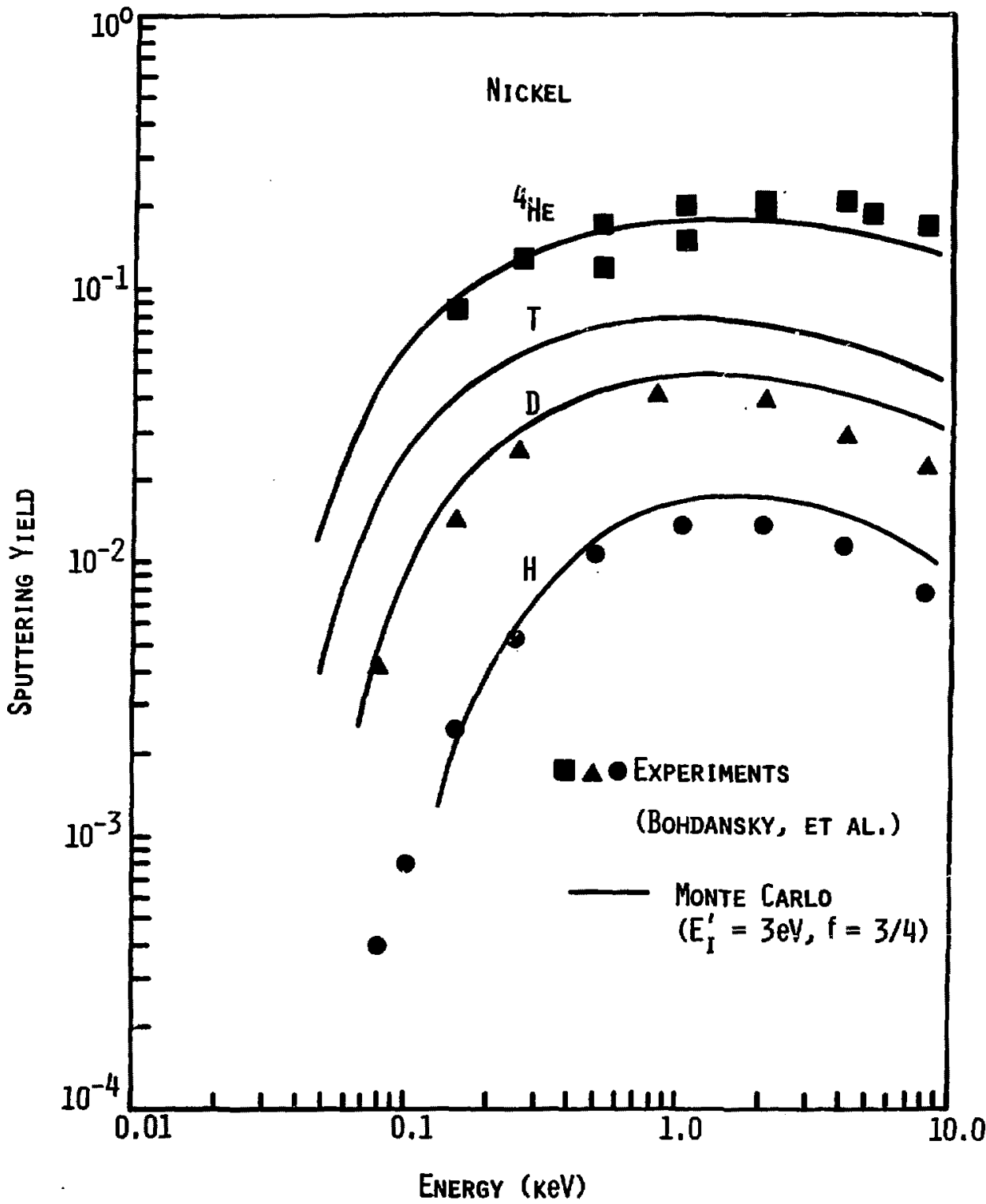

Figure 2 


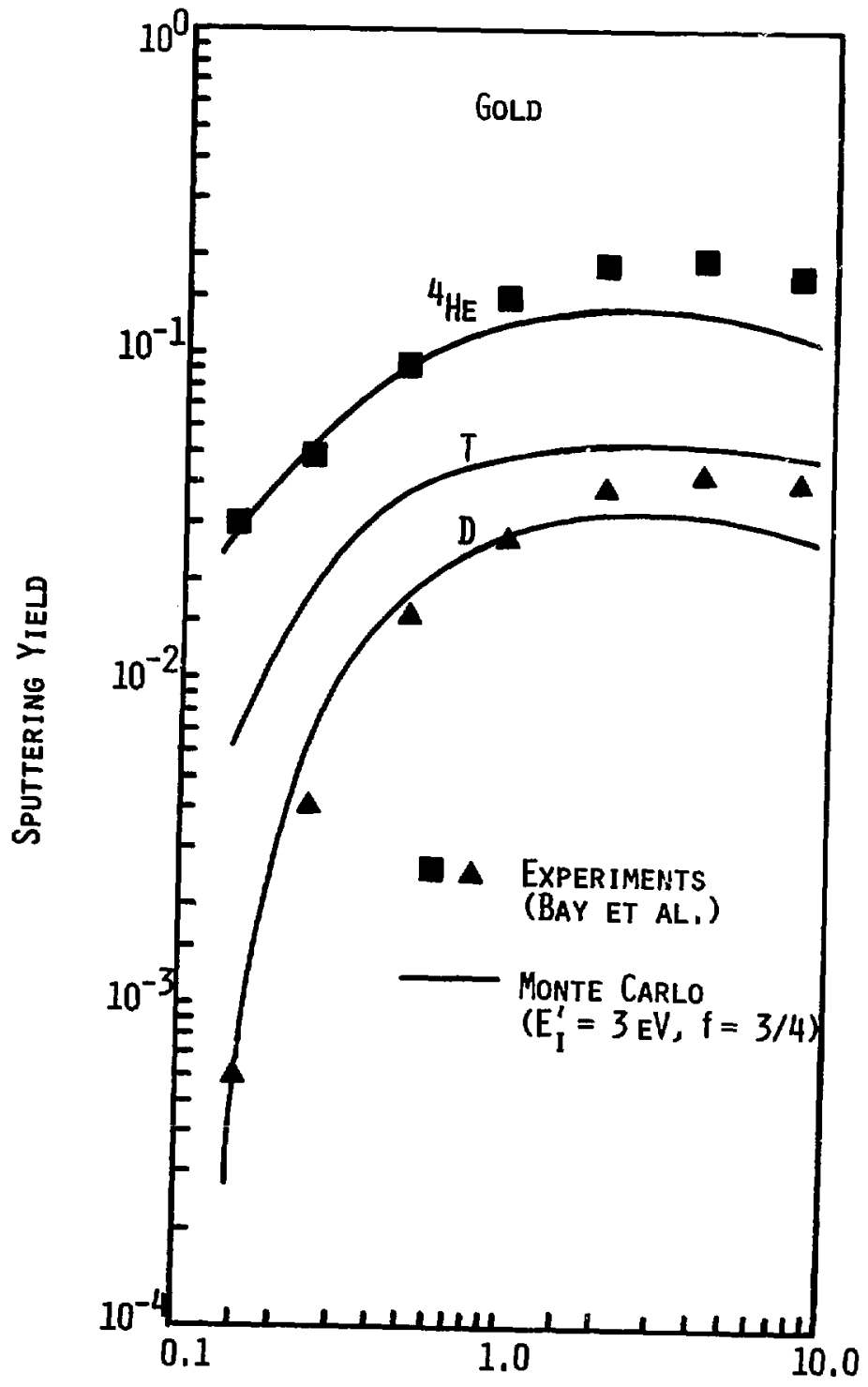

Energy (KeV)

Figure 3 


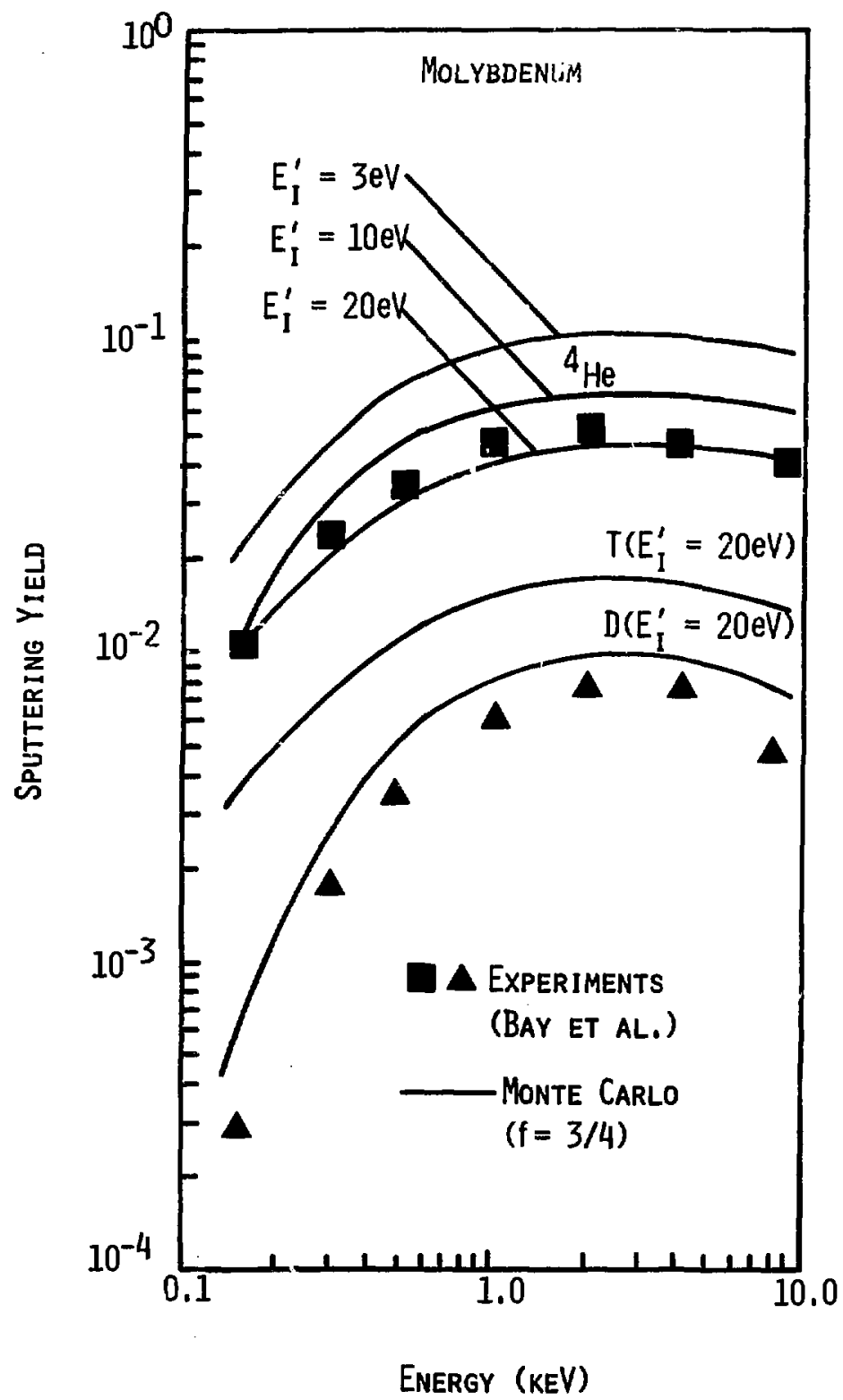

Figure 4 


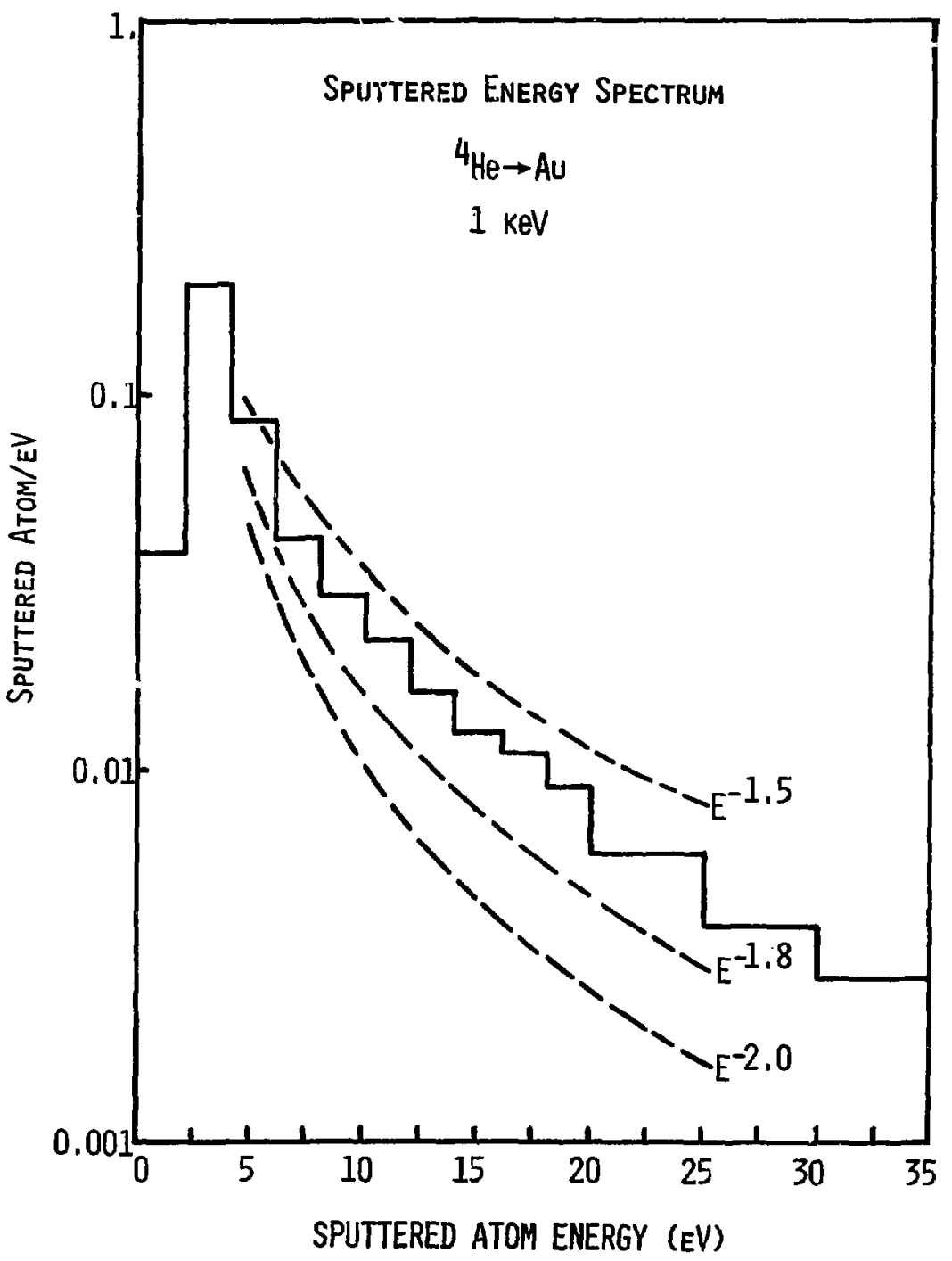

Figure 5 


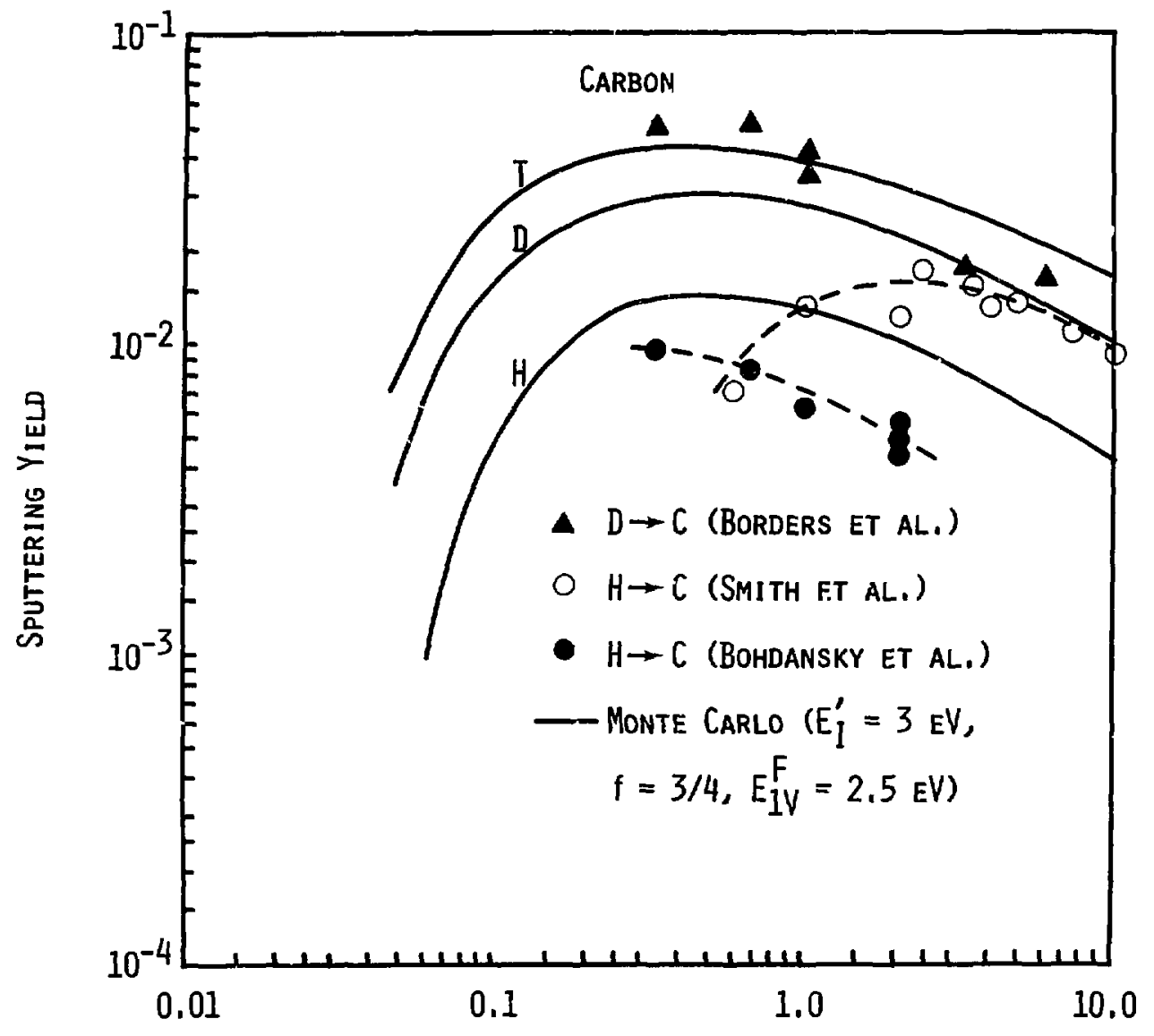

Energy (KeV)

Figure 6 